University of Nebraska - Lincoln

DigitalCommons@University of Nebraska - Lincoln

Faculty Publications, Department of Psychology

Psychology, Department of

March 1998

\title{
Differentiating Withdrawal Patterns Between Smokers and Smokeless Tobacco Users
}

Dennis E. McChargue

University of Nebraska-Lincoln, dmcchargue2@unl.edu

Frank L. Collins Jr.

Oklahoma State University

Follow this and additional works at: https://digitalcommons.unl.edu/psychfacpub

Part of the Psychiatry and Psychology Commons

McChargue, Dennis E. and Collins, Frank L. Jr., "Differentiating Withdrawal Patterns Between Smokers and Smokeless Tobacco Users" (1998). Faculty Publications, Department of Psychology. 272.

https://digitalcommons.unl.edu/psychfacpub/272

This Article is brought to you for free and open access by the Psychology, Department of at DigitalCommons@University of Nebraska - Lincoln. It has been accepted for inclusion in Faculty Publications, Department of Psychology by an authorized administrator of DigitalCommons@University of Nebraska - Lincoln. 
Published in Experimental and Clinical Psychopharmacology 6:2 (1998), pp. 205-208.

Copyright 1998 by the American Psychological Association, Inc. Used by permission.

"This article may not exactly replicate the final version published in the APA journal. It is not the copy of record."

\title{
Differentiating Withdrawal Patterns Between Smokers and Smokeless Tobacco Users
}

\author{
Dennis E. McChargue and Frank L. Collins, Jr. \\ Oklahoma State University \\ Correspondence: Frank L. Collins, Jr., Department of Psychology, Oklahoma State University, \\ 215 North Murray, Stillwater, Oklahoma 74078-3064; email: franklc@okway.okstate.edu.
}

\begin{abstract}
The present study was designed to document smokeless tobacco withdrawal patterns and to examine differential withdrawal responses between smokers and smokeless users. Participants $(\mathrm{N}=19)$ were studied under deprivation and nondeprivation conditions, 1 condition per week. The Withdrawal Symptoms Checklist was administered to assess cognitive and affective changes. Both smokers and smokeless users experienced substantially more withdrawal at 48-hr deprivation compared to the 48-hr nondeprivation condition. Participants in both groups endorsed decreasingly fewer withdrawal symptoms from $0 \mathrm{hr}$ to 48 $\mathrm{hr}$ on nondeprivation days. This downward trend suggests a need for stabilizing withdrawal responses before deprivation.
\end{abstract}

Smokeless tobacco use has increased by $38.4 \%$ over the past decade, while other nicotine products have progressively decreased (U.S. Department of Agriculture [USDA], 1993). Among the estimated 10 million American smokeless users, over one third of them are below the age of 21 (Consensus Conference, 1986). Epidemiological studies have also shown a relationship between smokeless use and oral cancers (Hoffmann, Adams, Lisk, Fisenne, \& Brunnemann, 1987; Hoffmann et al., 1995). Smokeless tobacco use at younger ages and the health risks involved with use necessitates more research examining smokeless tobacco effects (e.g., withdrawal).

Tobacco-withdrawal research has primarily focused on deprivation effects of smokers (Hughes \& Hatsukami, 1986; Hughes, Higgins, \& Hatsukami, 1990). Deprivation effects have been shown to begin within $24 \mathrm{hr}$ and peak within $48 \mathrm{hr}$ when compared to a baseline (prequit) period using self-report and physiological measurements (Hughes, Gust, Skoog, Keenan, \& Fenwick, 1991; Hughes \& Hatsukami, 1986). Smokeless tobacco studies have demonstrated comparable baseline cotinine levels (Gritz, Baer-Weiss, Benowitz, Van Vanakis, \& Jarvik, 1981; Hatsukami, Gust, \& Keenan, 1987) with subsequent deprivation producing quantitatively less severe withdrawal (Hatsukami et al., 1987). It was speculated that the differences in withdrawal severity may have been influenced by differential absorption rates, carbon monoxide effects, anticipatory effects, or all of these (Hatsukami et al., 1987).

Studies examining withdrawal patterns have consistently used 2 or 3 days of ad lib nicotine use before deprivation (Hatsukami et al., 1987; Hughes \& Hatsukami, 1986; Hughes et al., 1990). Although this research suggests the importance of stabilizing nondeprivation responses before deprivation (Hatsukami et al., 1987), researchers have not clearly documented that multiple nondeprivation days effectively stabilize the response pattern and control for demand characteristics. It is still unclear whether other factors, such as novelty or anticipation, significantly influence deprivation effects. For example, Hatsukami et al. speculated that the anticipation of prolonged abstinence may have contributed to cigarette smokers' higher withdrawal severity compared to smokeless users. Hatsukami et al. used smokers who enrolled in a treatment program and who intended to abstain permanently from smoking, whereas smokeless users expected to abstain for only $48 \mathrm{hr}$. This motivational difference may have influenced the results. Future research should focus on controlling for these effects. It is suggested that by counterbalancing nondeprivation and deprivation days we may get a clearer understanding of the stabilization process, help minimize novelty and anticipatory effects, and elicit a more accurate index of tobacco withdrawal.

This study addressed differences between cigarette smokers' and smokeless users' total withdrawal symptoms, withdrawal based on the American Psychiatric Association's Diagnostic and Statistical Manual of Mental Disorders, 4th edition (DSM-IV; American Psychiatric Association, 1994) criteria, and craving scores during nondeprivation and deprivation conditions. Consistent with previous research (Hatsukami et al., 1987; Hughes \& Hatsukami, 1986; Hughes et al., 1990; Keenan, Hatsukami, \& Anton, 1989), it was predicted that smokeless users and cigarette smokers would show significant increases in withdrawal and craving at 24-hr and 48-hr deprivation compared to a $0-\mathrm{hr}$ (prequit) baseline. Furthermore, it was expected that smokeless users and cigarette smokers would endorse substantially higher withdrawal and craving symptoms during the deprivation condition compared to the nondeprivation condition. Potential differences in withdrawal severity between smokeless users and cigarette smokers will also be examined.

\section{Method}

\section{Participants}

Nineteen male (10 smokeless, 9 smokers) undergraduate students participated in the study. The participants' ages ranged 
from 18 to 21 years. Thirteen participants were recruited from introductory psychology courses at Oklahoma State University and were given extra credit in their psychology classes as compensation for their participation. Six participants were volunteers from the campus population.

Participants included in the study were at least 18 years old, reported at least 1 year of continuous and daily nicotine use, were not currently attempting to stop or cut down on smoking or smokeless use, and did not report a history of multiple forms of nicotine use (e.g., smokes and uses smokeless tobacco). Smoking participants used an average of $13.9(S D=6.8)$ cigarettes per day, consumed $4.8(S D=2.4)$ packs per week, and averaged $5.2(S D$ $=2.9$ ) years of continuous use. Smokeless tobacco users on average reported using moist snuff products continuously for 4.0 ( $S D$ $=1.7)$ years, took $4.7(S D=1.2)$ dips per day, and consumed 2.9 $(S D=1.0)$ tins per week.

\section{Materials}

The Withdrawal Symptom Checklist (WSC) is a 12-item checklist structured in a Likert-scale format $(0=$ not present; 1 = mild; 2 = moderate; 3 = severe). This checklist was developed and standardized by Hughes and Hatsukami (1986) to assess nicotine withdrawal. Several symptoms detail a range of nicotine withdrawal criteria in the DSM- $I V$. Extracted $D S M-I V$ criteria allow for the comparison between total nicotine withdrawal and $D S M-I V$ nicotine withdrawal severity. The instrument has been shown to be valid and reliable (Hughes \& Hatsukami, 1986).

\section{Procedure}

Informed consent was obtained from all participants. Cigarette smokers (CIG) and smokeless tobacco (SMT) participants were assigned to deprivation conditions and nondeprivation conditions. Both conditions were counterbalanced. Five of the 10 SMT participants and 4 of the 9 CIG participants were assigned to the deprivation condition first and the nondeprivation condition second. During the deprivation condition, CIG and SMT participants were instructed to abstain from smoking or smokeless tobacco use for $48 \mathrm{hr}$, respectively. During the nondeprivation condition, CIG participants were instructed to maintain their smoking behavior for $48 \mathrm{hr}$, and SMT participants were instructed to maintain their smokeless tobacco use without increasing or decreasing the amount of consumption for $48 \mathrm{hr}$.

Data was collected at $0 \mathrm{hr}$ (prequit), $24 \mathrm{hr}$, and $48 \mathrm{hr}$ during each condition. All participants used their preferred nicotine product for $10 \mathrm{~min}$ before the administration of the WSC at $0 \mathrm{hr}$. Subsequent WSC assessments were taken $24 \mathrm{hr}$ and $48 \mathrm{hr}$ after the 0 -hr assessment period.

A carbon monoxide (COa) level was also taken during 0-hr, 24-hr, and 48-hr deprivation and nondeprivation assessment periods. Although COa levels do not detect smokeless tobacco use, CIG and SMT participants were told that COa levels would identify nicotine in the system. COa levels represented a bogus pipeline for the SMT users and provided a measure of smoking behavior for CIG participants. CIG participants were required to score above 8 ppm during the nondeprivation condition and below 4 ppm during the deprivation condition. COa assessments were implemented to increase compliance rates for all participants. Participants were debriefed following the last assessment period.

\section{Results}

Total withdrawal symptoms on 12 specific withdrawal symptoms were computed for each participant during all 3 deprivation and nondeprivation days. Craving scores and six DSM-IV withdrawal criteria items (i.e., irritability, anxiety, difficulty concentrating, restlessness, hunger, and insomnia) were extracted from the participants' reported total withdrawal symptoms. Mixed design analyses of variance (ANOVAs) comparing repeated measures of time ( $0 \mathrm{hr}, 24 \mathrm{hr}, 48 \mathrm{hr})$ and condition (deprivation vs. nondeprivation) with group (CIG vs. SMT) were conducted on $D S M-I V$ criteria withdrawal scores, total withdrawal scores, and craving scores.

Total withdrawal, DSM-IV withdrawal, and craving did not produce significant findings for the Group $\times$ Condition $\times$ Time, Group $\times$ Condition, Group $\times$ Time, or Group comparisons. Significant differences for the Condition $\times$ Time interaction were found for total withdrawal, $F(2,34)=8.13, p<.001, D S M-I V$ withdrawal, $F(2,34)=11.29, p<.0001$, and craving, $F(2,34)=$ $12.41, p<.0001$ (see Figure 1).

Tukey comparisons demonstrated significant total withdrawal differences between 48-hr deprivation and 48-hr nondeprivation for all three measures. DSM-IV and craving scores showed similar differences comparing deprivation and nondeprivation days at $24 \mathrm{hr}$. All significant differences were found at the $p<.05$ alpha level.

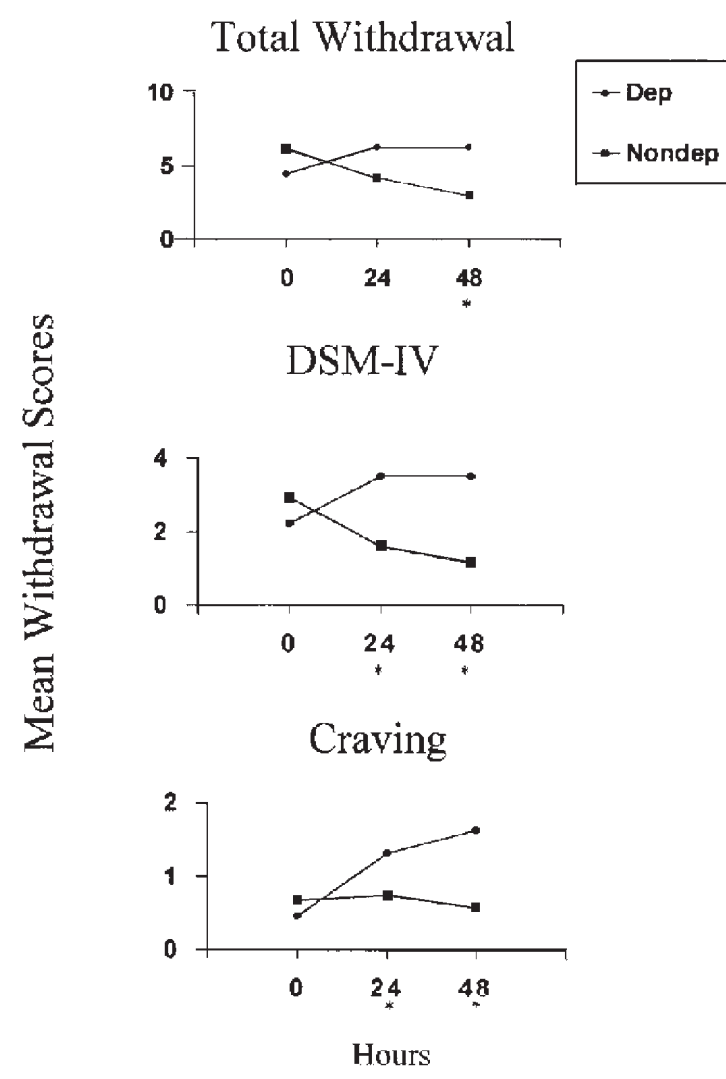

Figure 1. Mean withdrawal differences between deprivation and symptoms were computed for each participant during all 3 nondeprivation conditions for smokers and smokeless users. 
Mean deprivation and nondeprivation COa levels were calculated for both CIG and SMT participants. CIG participants' COa nondeprivation levels were $11.7 \mathrm{ppm}(S D=5.3)$ at baseline, $14.4 \mathrm{ppm}(S D=7.4)$ at $24 \mathrm{hr}$, and $12.8 \mathrm{ppm}(S D=5.4)$ at $48 \mathrm{hr}$. CIG participants' COa deprivation levels were $13.0 \mathrm{ppm}(S D=$ 6.7) at baseline, $2.9 \mathrm{ppm}(S D=0.9)$ at $24 \mathrm{hr}$, and $2.4 \mathrm{ppm}(S D=$ $1.0)$ at $48 \mathrm{hr}$. SMT participants' COa nondeprivation levels were $0.2 \mathrm{ppm}(S D=0.4)$ at baseline, $0.3 \mathrm{ppm}(S D=0.7)$ at $24 \mathrm{hr}$, and $0.2 \mathrm{ppm}(S D=0.4)$ at $48 \mathrm{hr}$. SMT participants' deprivation COa levels were $0.2 \mathrm{ppm}(S D=0.4)$ at baseline, $0.1 \mathrm{ppm}(S D=0.3)$ at $24 \mathrm{hr}$, and $0.3 \mathrm{ppm}(S D=0.5)$ at $48 \mathrm{hr}$.

\section{Discussion}

The results of this study partially supported the overall hypotheses. Nicotine users, regardless of nicotine type, showed significant increases in their withdrawal and craving symptoms as a function of 48-hr deprivation compared to 48-hr nondeprivation. Unexpectedly, smokeless users and cigarette smokers did not report substantial increases in withdrawal when compared to a $0-\mathrm{hr}$ prequit day. Consistent with previous research (Hatsukami et al., 1987), however, there were no significant group differences between smokeless users' and cigarette smokers' withdrawal. Furthermore, the reported quantitative differences in withdrawal of cigarette smokers compared to smokeless users, which was documented by Hatsukami et al. from observed differences in significant alpha levels (smokers, $p<.01$; smokeless users, $p<.05$ ), was not demonstrated in this study.

This study attempted to provide an alternative method of measuring withdrawal symptoms and severity. Similar to the Hatsukami et al. (1987) study comparing smokers' and smokeless users' withdrawal, our study attempted to extend this research by replicating the comparison of $0-\mathrm{hr}, 24-\mathrm{hr}$, and 48-hr deprivation days. It is still unclear in other withdrawal research, however, whether the typical 2 or 3 ad lib using days adequately stabilizes the response pattern of repeated measures before deprivation. Unlike Hatsukami et al. and other studies, our study attempted to provide a control for deprivation effects by creating the ad lib using days into a distinct $0-\mathrm{hr}, 24-\mathrm{hr}$, and 48-hr nondeprivation condition that mimics the deprivation methodology and by counterbalancing this condition with the standard deprivation condition. The results demonstrated that one baseline measure ( 0 -hr prequit) compared to deprivation effects does not accurately reflect withdrawal responsivity. As seen in Figure 1, a nonsignificant downward trend during nondeprivation days supported the idea of stabilizing response patterns before deprivation. This trend suggests that other effects may contaminate deprivation effects without an adequate control.

Although other studies have used nondeprivation days to stabilize response patterns (Hatsukami et al., 1987; Hughes \& Hatsukami, 1986; Hughes et al., 1990), this study is the first to examine the importance of stabilization. The most robust findings occurred when we compared 48-hr nondeprivation to 48 -hr deprivation effects. These findings suggest that several baseline measures are important for stabilizing response patterns before deprivation. Establishing 3 or more days of ad lib use, similar to Hatsukami et al., or using our methodology appears to elicit expected results. Our methodology, however, examines both nondeprivation and deprivation effects in a more controlled fashion.
Our results also extended nicotine-withdrawal research by comparing DSM-IV, craving, and total withdrawal severity. Previous studies have typically compared total withdrawal severity and craving (Hatsukami et al., 1987; Hughes \& Hatsukami, 1986; Hughes et al., 1990). The examination of DSM-IV symptoms compared to total withdrawal and craving adds support to differential response patterns that contribute to our understanding of diagnosable nicotine withdrawal from general withdrawal. For example, $D S M-I V$ withdrawal criteria demonstrated a quantitatively shorter latency in severe withdrawal responses compared to total withdrawal. This effect was evinced by the significant $D S M-I V$ differences in 24-hr deprivation compared to 24-hr nondeprivation comparisons. Total withdrawal comparisons lacked this effect.

There are two possible limitations to this study. First, documented withdrawal in smokers and smokeless users was minimal compared to other studies. However, these minimal findings may be a function of controlled novelty or anticipatory effects provided by the study's methodology. Second, comparisons across products are difficult to interpret because differences in the extent of nicotine exposure may influence withdrawal. This limitation is indicative of the importance in examining smokeless tobacco withdrawal. Previous research (Hatsukami et al., 1987) and this study have also provided evidence that this initial comparison is an important endeavor toward understanding smokeless tobacco withdrawal, Future research should refine the investigation of smokeless tobacco withdrawal by possibly examining potential adjuncts specific to smokeless tobacco use and exposure that may influence withdrawal.

These results emphasize two important considerations with the study of nicotine withdrawal. First, smokeless tobacco users do not appear to experience less intense withdrawal patterns. It has been speculated that smokeless users were a less dependent population partially as a result of fewer withdrawal symptoms (Hatsukami et al., 1987). Our findings, however, indicate the importance of future research with this population. Second, repeated measures may bias deprivation effects by using only baseline comparisons. Although previous research utilized 3 nondeprivation days before deprivation (Hatsukami et al., 1987), our study is the first to systematically examine the importance of stabilization.

\section{References}

American Psychiatric Association. (1994). Diagnostic and statistical manual of mental disorders (4th ed.). Washington, DC: Author.

Consensus Conference. (1986). Health applications of smokeless tobacco. Journal of the American Medical Association, 255, 1045-1048.

Gritz, E. R., Baer-Weiss, V., Benowitz, N. L., Van Vanakis, H., \& Jarvik, M. E. (1981). Plasma nicotine and cotinine concentrations in habitual smokeless tobacco users. Clinical Pharmacology Therapeutics, 30, 201-209.

Hatsukami, D., Gust, S. W., \& Keenan, R. M. (1987). Physiologic and subjective changes from smokeless tobacco withdrawal. Clinical Pharmacology and Therapeutics, 41, 103-107. 